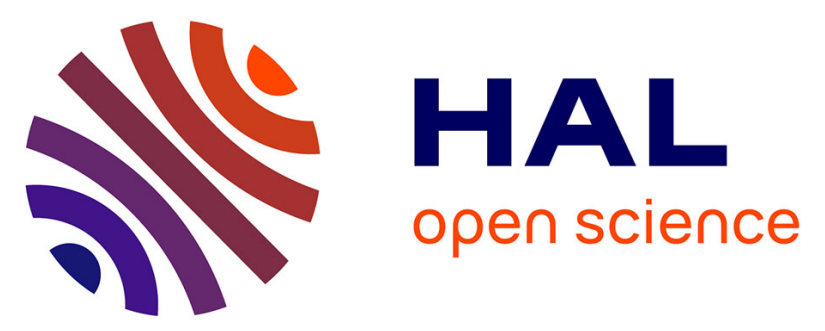

\title{
Les politiques de partage de données des financeurs d'essais cliniques en France
}

Pauline Rollando, Céline Parc, Florian Naudet, Jeanne Fabiola Gaba

\section{To cite this version:}

Pauline Rollando, Céline Parc, Florian Naudet, Jeanne Fabiola Gaba. Les politiques de partage de données des financeurs d'essais cliniques en France. Therapies, 2020, 75 (6), pp.527-536. 10.1016/j.therap.2020.04.001 . hal-02862266

\section{HAL Id: hal-02862266 https://hal-univ-rennes1.archives-ouvertes.fr/hal-02862266}

Submitted on 15 Jun 2020

HAL is a multi-disciplinary open access archive for the deposit and dissemination of scientific research documents, whether they are published or not. The documents may come from teaching and research institutions in France or abroad, or from public or private research centers.
L'archive ouverte pluridisciplinaire HAL, est destinée au dépôt et à la diffusion de documents scientifiques de niveau recherche, publiés ou non, émanant des établissements d'enseignement et de recherche français ou étrangers, des laboratoires publics ou privés. 


\section{THERAPIES}

\section{HEADING : Pharmacologie clinique}

\section{Les politiques de partage de données des financeurs d'essais cliniques en France}

\section{Data sharing policies of funders of clinical trials in France}

Politique de partage de données

\section{Pauline Rollando ${ }^{\mathrm{a}, *}$, Céline Parc ${ }^{\mathrm{b}}$, Florian Naudet ${ }^{\mathrm{a}}$, Jeanne Fabiola Gaba ${ }^{\mathrm{a}, \mathrm{c}}$}

${ }^{a}$ Univ Rennes, CHU Rennes, Inserm, CIC 1414 (centre d'investigation clinique de Rennes), 35000 Rennes, France

${ }^{b}$ CHU Rennes, DRI (Direction de la recherche et de l'innovation), 35000 Rennes, France

${ }^{c}$ REPERES (REcherche en Pharmaco-Epidémiologie et REcours aux Soins), 35000 Rennes, France

Reçu le 11 octobre 2019 ; accepté le 24 février 2020

*Auteur correspondant. Centre d'investigation clinique, CIC 1414, CHRU Rennes, 2, rue Henri Le Guilloux, 35000 Rennes, France.

Adresse e-mail : pau.rollando@gmail.com (P. Rollando)

\section{Summary}

Aims.- The aims of this survey were to evaluate the percentage of French clinical trial funders with a data sharing policy, to describe their data sharing policies and, more generally, the transparency of the research they fund. Methods.- The different funders of clinical trials in France have been identified from 3 lists of tenders for clinical research projects: the internal list of the University Hospital Center (CHU) of Rennes, the list of the Interregional Group for Clinical Research and Innovation (GIRCI EST), the list of the portal for calls for projects in health research. Funders were contacted, first by email and then by phone (at least two email and / or phone reminders) to respond to an online survey via Google form. The questionnaire aimed to assess the existence of a sharing policy or not, as well as the way in which it was set up. Results.- Out of 190 funders contacted, 94 did not respond. 65 of the respondents were excluded because they did not fund clinical trials. Of the 31 funders included (including 
Direction générale de l'offre de soins [DGOS], Institut national contre le cancer [INCa], Groupement Interrégional de Recherche Clinique et d'Innovation [GIRCIs]), only 9 (29\%) had implemented a data sharing policy. Among these nine funders, only one had a mandatory sharing policy and eight a policy supporting but not enforcing data sharing. Five allowed the use of budget lines dedicated to data sharing. Three reported granting data sharing incentives. Three had dedicated guidelines indicating a specific mode of sharing data (sharing on request and / or on a specialized platform) and specifying the type of data (individual patient data and / or protocol and amendments). For all three, there were restrictions on sharing data to researchers only. Data sharing policies concerned $19 \%$ of the total financial volume (850,032,000 euros) of the 26 financiers who reported this information. Conclusion.- Despite international interest in clinical trial data sharing practices, clinical trials funders with a strong data-sharing policy remain an exception in France.

Keywords: Clinical trial; Data sharing; Transparency of research 


\section{Résumé}

Objectifs.- Les objectifs de cette enquête étaient d'évaluer le pourcentage de financeurs d'essais cliniques français ayant une politique de partage de données et de décrire les grandes lignes de leurs politiques en matière de partage de données et, de manière plus générale, de transparence de la recherche. Méthodes.- Les différents financeurs d'essais cliniques en France ont été identifiés à partir de 3 listes d'appels d'offres de projet de recherche clinique : la liste interne du centre hospitalier universitaire (CHU) de Rennes, la liste du Groupement interrégional de recherche clinique et d'innovation (GIRCI EST), la liste du portail des appels à projets de la recherche en santé. Les financeurs étaient contactés, d'abord par email puis par téléphone (au moins deux relances email et/ou téléphoniques) afin de répondre à un sondage en ligne via Google form. Le questionnaire visait à évaluer l'existence d'une politique de partage ou non, ainsi que la manière dont elle était mise en place. Résultats.- Sur 190 financeurs contactés, 94 n’ont pas répondu et 65 ont été exclus car ne finançaient pas d'essais cliniques. Sur les 31 financeurs inclus (parmi lesquels la Direction générale de l'offre de soins [DGOS], l'Institut national contre le cancer [INCa], les Groupements interrégionaux de recherche clinique et d'innovation [GIRCIs]), seuls 9 (29\%) avaient mis en place une politique de partage de données issus d'essais contrôlés randomisés (ECR) financés. Parmi ces neuf financeurs, un seul avait une politique rendant le partage obligatoire et huit une politique encourageant le partage. Cinq permettaient l'utilisation de lignes budgétaires dédiées au partage des données. Trois déclaraient octroyer des incitatifs au partage des données. Trois avaient des guidelines dédiées orientant vers un mode spécifique de partage de données concernant le modèle de partage (partage sur requête et/ou sur une plateforme spécialisée) et le type de données (données individuelles des patients et/ou protocole et amendements). Pour les trois, il y avait des restrictions vers un partage de données aux chercheurs uniquement. Le partage des données concernait $19 \%$ du volume financier total (850 032000 euros) des 26 financeurs nous ayant 
déclaré cette information. Conclusion.- Malgré l'intérêt au plan international pour les pratiques de partage des données d'essais cliniques, les financeurs ayant une réelle politique de partage de données restent une exception en France.

\section{MOTS CLÉS}

Essai clinique ; Partage de donnée ; Transparence de la recherche 


\section{Abréviations}

ANR : Agence nationale de la recherche

AO : appels d'offres

APICIL : Association de retraites complémentaires pour l'industrie et le commerce lyonnais

ARSEP : Aide à la recherche contre la sclérose en plaques

ARSLA : Association pour la recherche sur la sclérose latérale amytrophique

BMJ : British Medical Journal

CHU : centre hospitalier universitaire

CIC : centre d'investigation clinique

CNRS : Centre national de la recherche scientifique

DGOS : Direction générale de l'offre de soins

DIP : données individuelles des patients

ECR : essai contrôlé randomisé

EFPIA: European Federation of Pharmaceutical Industries and Associations

GIRCI : Groupement interrégional de recherche clinique et d'innovation

ICMJE: International Committee of Medical Journal Editors

INCa : Institut national du cancer

INSERM : Institut national de la santé et de la recherche médicale

$\mathrm{PD}$ : politique de partage

PHRC : programme hospitalier de recherche clinique

PHRC-I : programme hospitalier de recherche clinique inter-régional

PHRC-K : programme hospitalier de recherche clinique en cancérologie

PHRC-N : programme hospitalier de recherche clinique national

PLOS: Public Library Of Science

\section{Introduction}


Les promesses d'un partage responsable des données issues des essais contrôlés randomisés (ECR) sont majeures avec la possibilité de réaliser des méta-analyses sur données individuelles, des analyses secondaires et des réanalyses. Des initiatives par des journaux scientifiques internationaux comme le British Medical Journal (BMJ) ou Public Library Of Science Medicine (PLOS) [1] ont été entreprises, afin de faire du partage des données des essais randomisés une réalité. En juin 2017, l'International Committee of Medical Journal Editors (ICMJE) a défini de nouvelles règles en matière de partage de données [2] et incite les chercheurs à spécifier un plan de partage des données dans leurs publications (c'est-à-dire à préciser les modalités fixées par la politique ICMJE pour un partage éventuel des données).

Au-delà de ceux qui publient la recherche, ceux qui la financent ont un rôle clef dans l'établissement de cette nouvelle norme. Certaines firmes pharmaceutiques ont développé des politiques de partage en mettant en ligne les données de leurs essais sur des plateformes comme la clinical study data request [3]. D'importants bailleurs de fonds de la recherche clinique comme le Wellcome Trust, impose aux chercheurs qu'ils financent, de rendre disponible les données de recherche nécessaires à la réplication des analyses au moment de la publication [4].

Face à ces nouveaux standards internationaux, qu'en est-il des financeurs d'essais cliniques en France ? Plusieurs associations de patients, des syndicats de médecins et Médecins du monde ont lancé un appel à l'Institut national de la santé et de la recherche médicale (INSERM) et au Centre national de la recherche (CNRS) pour améliorer la transparence autour des ECR [5]. Un plan national de science ouverte a été lancé le 4 juillet 2018 par le ministère de l'Enseignement supérieur, de la Recherche et de l'Innovation [6] visant, entre autre, à rendre obligatoire la diffusion ouverte des données de recherche issues de programmes financés par appels à projets sur fonds publics. Dans ce contexte, les objectifs de cette enquête étaient d'évaluer le pourcentage de financeurs français ayant une politique de partage de données parmi 
ceux sélectionnés et de décrire les grandes lignes de leurs politiques en matière de partage de données et, de manière plus générale, de transparence de la recherche.

\section{Méthodes}

Nous avons enregistré le protocole avant le début de cette enquête sur l'Open Science Framework [7].

Les différents financeurs français ont été sélectionnés à partir de 3 listes d'appels d'offres de projet de recherche clinique distincts :

- la liste interne du centre hospitalier universitaire (CHU) de Rennes. La direction de la recherche clinique du CHU de Rennes tient une liste à jour des principaux appels à projets en recherche clinique et la diffuse auprès des enseignant-chercheurs du CHU ;

- la liste du Groupement interrégional de recherche clinique et d'innovation (GIRCI EST), qui est un portail commun aux 7 GIRCI répertoriant les informations sur les appels à projets de recherche en santé avec un moteur de recherche intégré permettant de faire le tri entre les subventions de recherche, les bourses postdoctorales, doctorales, de Master et les prix récompensant des travaux réalisés.

- la liste du portail des appels à projets de la recherche en santé, qui rassemble l'ensemble des appels à projets publics dans les domaines de la recherche translationnelle, clinique, et en santé publique. Il réunit neuf partenaires, lanceurs d'appel à projets de recherche dans ces domaines.

À partir de ces trois listes d'appels d'offres de projet de recherche clinique, nous avons inclus les financeurs proposant des subventions de recherches en France. Les financeurs proposant uniquement des bourses et/ou des prix et/ou du financement de matériel n'étaient donc pas inclus. Les financeurs n'ayant pas de site web, ni de numéro de téléphone joignable, ni de contact e-mail référencié n'étaient pas inclus. 
Après un premier contact avec ces financeurs (ou en fonction des informations présentées sur leur site internet), étaient exclus ceux qui ne finançaient pas d'ECR. Au cours de l'étude, nous avons dû ajouter les critères d'exclusion suivant car d'évidence non adaptés : les universités, ambassades, et les intermédiaires entre chercheurs et financeurs. Enfin, lorsqu'un financeur était identifié via différents appels à projets, il n'était compté qu'une fois.

L'étude a été menée entre le 04 Avril 2019 (date de l'envoi du premier mail) et le 17 juin 2019 (date de la dernière réponse). Les financeurs étaient contactés, d'abord par email puis par téléphone (au moins deux relances par email et/ou téléphoniques) afin de répondre à un sondage en ligne via Google form. Le questionnaire visait à évaluer les critères de jugement principal et secondaires. Le critère principal était l'existence d'une politique de partage. Lorsqu'une politique de partage était présente, les critères de jugement secondaires étaient : le type de politique de partage (politique rendant le partage obligatoire ou politique encourageant le partage), l'existence de lignes budgétaires dédiées au partage des données, l'existence d'un autre incitatif au partage des donnée (et, si oui, le type d'incitatif), l'existence de sanctions en cas de non-partage (et, si oui, quel type de sanctions ), l'existence de guidelines pour un mode spécifique de partage de données (et, si oui, quel type de partage, c'est-à-dire partage sur requête ou sur différents types de plateforme), le type de données à partager (les données individuelles, le code statistique, et toute autre type de données), l'existence de restrictions vers un partage de données aux chercheurs uniquement. Le tableau 1 présente plus clairement ces différentes modalités.

Au-delà des critères portant sur le partage des données, d'autres aspects de leurs politiques étaient évalués pour tous les financeurs à savoir l'obligation d'un enregistrement préalable des recherches et l'existence d'incitatifs à la publication des résultats de l'étude (quels qu'étaient les résultats de l'étude). 
Enfin, le sondage précisait les caractéristiques du financeur à savoir le nombre d'appels d'offres du financeur sur l'année 2018, le volume financier annuel (2018) de l'ensemble des appels d'offres de ce financeur.

Les réponses au questionnaire ont été rapportées et décrites sous forme d'effectifs, de pourcentages, de médiane (et intervalles interquartiles). Les analyses statistiques ont été réalisées à l'aide du logiciel $\mathrm{R}$ [8].

\section{Résultats}

La Fig. 1. représente les différentes étapes de sélection des financeurs d'ECR inclus dans notre enquête. Parmi les 276 financeurs identifiés, 86 ont été exclus. Sur les 190 contactés, le taux de réponse était de $51 \%$. Soixante-cinq des financeurs contactés et ayant répondu ont été finalement exclus car ils ne finançaient pas d'ECR. Nous avons alors inclus et analysé les réponses de 31 des financeurs contactés. La liste des financeurs analysés est présentée dans l'Annexe 1.

Le tableau 2 présente les caractéristiques descriptives des financeurs inclus selon l'implémentation d'une politique de partage ou non. Le volume financier sur l'année 2018, le nombre d'appels d'offres sur l'année 2018, réglementation sur l'enregistrement des ECR financés et la publication des résultats quel que soit le résultat sont les questions communes à tous les financeurs inclus. Sur les 31 financeurs inclus, les informations concernant le nombre d'appels d'offres et/ou le volume financier ne nous ont pas été communiquées pour 5 d'entre eux.

Parmi les 31 financeurs d'essais cliniques français inclus, seuls 9 (29\%) déclarent avoir une politique de partage de données avec une obligation d'enregistrement préalable des ECR pour six d'entre eux. Treize (42\%) financeurs déclarent rendre obligatoire la publication des résultats (qu'ils soient positifs ou négatifs), dont 6 ayant une politique de partage de données. 
Six $(27 \%)$ des financeurs n'ayant pas déclaré de politique de partage de données rendent obligatoire l'enregistrement préalable des ECR.

Le nombre d'appels d'offres médian en 2018 des financeurs inclus est de 2. Le volume financier médian des appels d'offres en 2018 est de $420000 €$ pour les financeurs ayant déclaré une politique de partage et de $1400000 €$ pour ceux n'en ayant pas déclaré. Les dix principaux financeurs en termes de volume financier parmi ceux inclus sont listés dans le tableau 3. La Fig. 2 présente la part en termes de nombre d'appels d'offres et de volume financier sur l'année 2018 des financeurs des essais cliniques qui déclarent avoir une politique de partage de données. Le partage des données concernait $19 \%$ du volume financier total (850 032000 euros) des 26 financeurs nous ayant déclaré cette information.

Le tableau 4 présente les caractéristiques des politiques de partage de données recueillies via le questionnaire. Trois financeurs ont déclaré avoir des guidelines orientant vers un mode spécifique de partage de données (le partage sur requête et/ou le partage via une plateforme spécialisée) avec une restriction du partage aux chercheurs uniquement. Les types de données disponibles au partage sont : les données individuelles des patients, le protocole et ses amendements. Cinq financeurs déclarent dédier des lignes budgétaires au partage de données dont 3 ont mis en place des incitatifs au partage. La Ligue contre le cancer est le seul financeur à avoir fourni un détail des incitatifs : une aide aux investigateurs pour l'organisation du recueil d'analyse de données au plus haut niveau scientifique à travers des financements de plateformes de recherche clinique. Pour un autre financeur, l'incitatif déclaré n'était pas clairement exprimé et nous n'avons pas pu avoir confirmation malgré plusieurs relances.

\section{Discussion}

Sur les 31 financeurs analysés, $29 \%$ des financeurs français avaient une politique de partage de données issues d'ECR. Plus précisément, parmi les 10 plus gros financeurs que nous avons 
pu identifier en termes de volume financier, un seul a déclaré faire du partage des données une possibilité encouragée.

Dans un échantillon international de 18 financeurs majeurs, 2 (11\%) avaient une politique imposant le partage et $9(50 \%)$ une politique incitant au partage [9]. Toute comparaison reste difficile, mais en tout état de cause, les politiques de partage de données obligatoires restent l'exception aussi bien à l'international qu'en France. Par contre, en ce qui concerne, les politiques encourageant au partage, celles-ci sont presque inexistantes en France alors qu'elles sont clairement plus développées à l'étranger.

Il est difficile d'obtenir une liste complète des financeurs de la recherche clinique en France. Pour avoir une liste plus exhaustive, les financeurs analysés dans notre étude ont été sélectionnés en croisant trois listes différentes d'appels d'offres majeures. Mais ces listes ne comprenaient pas que des financeurs d'essais randomisés contrôlés : il y avait des régions, des ambassades et des financeurs que bon nombre de chercheurs en recherche clinique n'ont pas l'habitude de contacter. La mise en place d'un registre listant les différents bailleurs de fonds de la recherche clinique en France pourrait être un plus pour une meilleure diffusion de l'information. Ce serait un moyen efficace pour un chercheur d'avoir des informations sur les possibles bailleurs de fonds pour sa recherche et les différentes politiques de ces derniers en matière de partage de données et toute autre mesure ayant pour but d'améliorer l'intégrité et la qualité de la recherche. Un exemple de ce type de registre est « Sherpa-Juliet » qui récence les conditions des bailleurs de fonds pour une publication en « open access » [10]. Il existe aussi des interfaces universitaires pour informer les chercheurs sur les recommandations des bailleurs de fonds ayant une politique de partage de données et open access [11].

Nous avons rencontré des difficultés à contacter les financeurs : pour certains nous n'avions aucunes coordonnées ni téléphoniques, ni mail et pour d'autres, nous n'avions pas reçu de réponses malgré nos relances (les personnes répondant au téléphone ou par mail ne 
sachant parfois pas nous orienter). Cependant, nous avons pu collecter des informations sur les principaux financeurs d'essais cliniques à savoir la Direction générale de l'offre de soins (DGOS), l'Institut national contre le cancer (INCa) et les différents Groupements interrégionaux pour la recherche clinique et l'innovation (GIRCI). Nous avons aussi pris en compte la réponse de l'ANR qui apparaît comme l'agence ayant le plus gros volume financier. Néanmoins, la part de la recherche clinique dans les financements de l'ANR est faible et assurément moins importante que celle de la DGOS qui s'occupe du lancement des programmes de recherche financés par le ministère des Solidarités et de la Santé. Le programme hospitalier de recherche clinique (PHRC) est l'un de ces programmes et il constitue l'un des plus importants financements de la recherche clinique en France. Le PHRC se décline en trois appels à projets : le programme hospitalier de recherche clinique national (PHRC-N), le programme hospitalier de recherche clinique en cancérologie (PHRC-K) dont la gestion est assurée par l'INCa, le programme hospitalier de recherche clinique inter-régional (PHRC-I) dont la gestion est assurée par chaque GIRCI. Le suivi des projets retenus s'appuie sur un découpage en phases de leur déroulement et conditionne leur financement, adapté à l'avancement de la recherche. En plus du PHRC, les GIRCI possèdent une enveloppe interne qui leur permet de financer des projets de recherche qui peuvent être de la recherche clinique.

Notre questionnaire a été construit pour récolter les informations sur les politiques de partage mais pouvait passer à côté de subtilités, que seule une approche plus qualitative pourrait mieux appréhender. Une approche plus qualitative aurait permis une vérification supplémentaire des réponses recueillies et aussi prendre en compte les décisions à venir des financeurs sur les notions de transparence et de réutilisation des données issues des essais cliniques. Par exemple s'il est clair que la DGOS n'a pas de politique de partage des données, elle n'est pas fermée à ces questions. Nos échanges avec ce financeur soulignent que des lignes de budget pour un partage de données pourraient être discutées au cas par cas. Indirectement, 
la politique de suivi des projets financés de la DGOS a pour but de permettre une meilleure visibilité et d'encourager le partage des résultats.

Les financeurs analysés dans notre étude sont pour la plupart des institutions gouvernementales, des associations et/ou des fondations dont les principales ressources financières proviennent de dons. Bien que notre étude montre une méconnaissance du partage de données dans le secteur public, il n'en est pas forcément le cas au niveau de certains industriels français (secteur pas pris en compte dans notre étude). Bien que politique de partage n'implique pas toujours un partage effectif, la Fédération européenne des industries et associations pharmaceutiques (EFPIA) a une politique de partage de données et les membres français suivent cette politique en rendant disponible les données issues des ECR et d'autres documents dans un but de transparence et de reproductibilité. De plus, l'agence Européenne du médicament a prévu de rendre obligatoire le partage avec sa politique 0070 de 2014 [12]. Cette prise de conscience de l'importance du partage de données doit être rependu au-delà de la réglementation des médicaments et être un des critères d'évaluation de la recherche clinique. Au-delà des financeurs, les initiatives de partage des données doivent impliquer les promoteurs et les responsables scientifiques des essais. Ceux-ci sont généralement considérés, à tort ou à raison, comme « propriétaires » de ces données et ont nécessairement un rôle important. Si les sondages à l'international, comme par exemple celui de Rathi et al. [13] révèlent généralement des intentions de partage élevées chez les investigateurs, il serait intéressant de réaliser un sondage, au plan français se focalisant sur les investigateurs et les différentes directions de la recherche clinique. Il serait intéressant d'y ajouter une partie qualitative explorant leurs perceptions en termes de valorisation de la recherche et leurs représentations sur le partage dans le contexte international actuel.

Mais, aucune initiative isolée ne saurait suffire. Par exemple, deux journaux d'envergure, le BMJ et PLOS Medicine ont imposé le partage des données pour les études 
randomisées qu'ils publiaient. La disponibilité des données n'était pas optimale mais le taux de partage des données de l'ordre de $50 \%$ était plus élevé qu'ailleurs dans la littérature biomédicale [1]. Les problèmes de contact avec les auteurs correspondants, le manque de ressources dans la préparation des ensembles de données et l'importante hétérogénéité des pratiques de partage des données sont autant d'obstacles à surmonter.

Le partage des données, est présenté par l'ICMJE comme un impératif d'ordre éthique [14]. En d'autres termes puisque les participants aux essais acceptent de prendre des risques, on leur doit en retour une utilisation optimale des données générées par la recherche. D’ailleurs, dans une étude américaine, les patients étaient dans une très large majorité favorable au partage de leurs données [15]. Le partage responsable des données maximiserait en effet les bénéfices que l'on peut attendre des essais en permettant des méta-analyses sur données individuelles, en permettant d'explorer des questions nouvelles sans avoir besoin de faire courir de nouveaux risques dans une nouvelle étude lorsqu'un jeu de données existe déjà et en permettant des réanalyses pour certaines études parfois controversées. Les financeurs sont assurément un levier supplémentaire pour faire du partage des données des essais thérapeutiques une réalité. Le partage de données n'est pas sans contraintes et les financeurs peuvent allouer les ressources manquantes aux équipes pour mettre en forme, anonymiser et transférer les données dans les conditions les plus sûres possibles. Au-delà d'imposer le partage, ils peuvent imposer qu'une information adaptée et claire sur ce partage soit donnée aux scientifiques et aux patients. Ils ont une responsabilité certaine à assurer une utilisation optimale des ressources qu'ils allouent et à éviter autant que possible le gaspillage des efforts de recherche. Bien entendu, les questions sont nombreuses quant au type et aux modalités des politiques à adopter. Au Royaume Uni, le Medical Research Council Hubs for Trials Methodology Research a proposé un modèle de partage des données individuelles sur requête raisonnable des études ayant reçu un financement public [16]. Au-delà, en faisant du partage des données, de la publication de tous les résultats, 
et d'autres pratiques de sciences ouvertes des critères servant à l'allocation des ressources, les financeurs pourraient contribuer à mieux aligner le système actuel d'incitatifs et de récompenses sur les besoins de la société et à valoriser la qualité des travaux scientifiques [17].

Nous pensons qu'il est temps de réunir les principaux financeurs et acteurs de la recherche clinique française pour définir d'une politique efficiente de partage, concrète, réfléchie et de mettre en place une évaluation des bénéfices apportées par ces politiques. Une telle politique de partage devra bien entendu prendre en compte la réglementation française relative à la protection des données et le règlement général sur la protection des données [18]. Elle devra aussi prendre en compte le positionnement des patients inclus dans les études pour lequels les attitudes vis-à-vis du partage sont positives [19]. Qui plus est toute politique devrait être associée une composante d'évaluation afin de voir si le partage mis en place assure les bénéfices attendus. L'enjeu est de maximiser la valeur du bien public inestimable que représentent les données de la recherche clinique.

\section{Remerciements}

Cette étude a été rendue possible grâce au soutien financier de Nationale de la Recherche (projet ReiTher, reproductibilité de la recherche thérapeutique, ANR-17-CE36-0010-01). Nous remercions également tous les participants qui ont bien voulu répondre à notre sondage ainsi que les personnes nous ayant donné accès aux listes de financeurs.

\section{Déclaration de liens d'intérêts}

Les auteurs n'ont pas de conflits d'intérêts à déclarer.

\section{Références}

[1] Naudet F, Sakarovitch C, Janiaud P, Cristea I, Fanelli D, Moher D, et al. Data sharing and reanalysis of randomized controlled trials in leading biomedical journals with a full data sharing policy: survey of studies published in The BMJ and PLOS Medicine. BMJ 2018;360:k400. 
[2] Taichman DB, Sahni P, Pinborg A, Peiperl L, Laine C, James A, et al. Data sharing statements for clinical trials: a requirement of the International Committee of Medical Journal Editors. JAMA 2017;317:2491-2. https://doi.org/10.1001/jama.2017.6514.

[3] Strom BL, Buyse ME, Hughes J, Knoppers BM. Data sharing - Is the juice worth the squeeze? N Engl J Med 2016;375(17):1608-9.

[4] Wellcome. Data, software and materials management and sharing policy. 2020. https://wellcome.ac.uk/funding/guidance/data-software-materials-management-andsharing-policy. [Consulté le 3 avril 2020].

[5] APMnews. L'Inserm et le CNRS appelés à améliorer la transparence entourant leurs essais cliniques. 2018. https://www.apmnews.com/nostory.php?uid=68878\&objet=328604. [Consulté le 3 avril 2020].

[6] Ministère de l'Enseignement supérieur, de la Recherche et de l'Innovation. Le plan national pour la science ouverte : les résultats de la recherche scientifique ouverts à tous, sans entrave, sans délai, sans paiement. 2018. http://www.enseignementsuprecherche.gouv.fr/cid132529/le-plan-national-pour-la-science-ouverte-les-resultats-de-larecherche-scientifique-ouverts-a-tous-sans-entrave-sans-delai-sans-paiement.html. [Consulté le 3 avril 2020].

[7] OSF. Politiques de partage de données des financeurs des essais cliniques randomisés en France. 2019. https://osf.io/mr5hx/. [Consulté le 3 avril 2020].

[8] R Core Team RF for SC. R: A Language and Environment for Statistical Computing. Vienna, Austria: 2018. https://www.r-project.org/. [Consulté le 3 avril 2020].

[9] DeVito NJ, French L, Goldacre B. Noncommercial funders' policies on trial registration, access to summary results, and individual patient data availability. JAMA 2018;319:1721-3.

[10] Research funders' open access policies - v2.sherpa. 2019. https://v2.sherpa.ac.uk/juliet/ [Consulté le 3 avril 2020]. 
[11] Nightingale A. Library: data management plans: Other major funder requirements. 2019. http://library.bath.ac.uk/research-data/data-management-plans/funderrequirements. [Consulté le 3 avril 2020].

[12] European Medecines Agency. European Medicines Agency policy on publication of clinical data for medicinal products for human use 2019. Mars 2019. https://www.ema.europa.eu/en/documents/other/european-medicines-agency-policypublication-clinical-data-medicinal-products-human-use_en.pdf. [Consulté le 3 avril 2020 (20 pp.)].

[13] Rathi V, Dzara K, Gross CP, Hrynaszkiewicz I, Joffe S, Krumholz HM, et al. Sharing of clinical trial data among trialists: a cross sectional survey. BMJ 2012;345:e7570.

[14] Taichman DB, Backus J, Baethge C, Bauchner H, De Leeuw PW, Drazen JM, et al. Editorial-sharing clinical trial data: a proposal from the International Committee of Medical Journal Editors. Ethiop J Health Sci 2016;26:2-4.

[15] Miller J, Ross JS, Wilenzick M, Mello MM. Sharing of clinical trial data and results reporting practices among large pharmaceutical companies: cross sectional descriptive study and pilot of a tool to improve company practices. BMJ 2019;366:14217.

[16] Sydes MR, Johnson AL, Meredith SK, Rauchenberger M, South A, Parmar MK. Sharing data from clinical trials: the rationale for a controlled access approach. Trials 2015;16:104.

[17] Moher D, Naudet F, Cristea IA, Miedema F, loannidis JPA, Goodman SN. Assessing scientists for hiring, promotion, and tenure. PLOS Biol 2018;16:e2004089.

[18] Demotes-Mainard J, Cornu C, Guérin A, Bertoye PH, Boidin R, Bureau S, et al. How the new European data protection regulation affects clinical research and recommendations? Therapies 2019;74:31-42.

[19] Mello MM, Lieou V, Goodman SN. Clinical trial participants' views of the risks and benefits of data sharing. N Engl J Med 2018;378:2202-11.

Tableau 1. Les différents éléments des politiques de partages pris en compte.

\begin{tabular}{|l|l|l|}
\hline $\begin{array}{l}\text { Critère de } \\
\text { jugement }\end{array}$ & Modalités & Explications \\
\hline
\end{tabular}




\begin{tabular}{|c|c|c|}
\hline \multirow[t]{2}{*}{$\begin{array}{l}\text { Type de } \\
\text { politique de } \\
\text { partage }\end{array}$} & Obligatoire & $\begin{array}{l}\text { Le partage des données est rendu obligatoire par le } \\
\text { financeur. Par exemple, la fondation Bill and Melinda } \\
\text { Gates impose que «les données ayant servi à produire } \\
\text { tous les résultats publiés seront accessibles et ouvertes } \\
\text { immédiatement » (https://www.gatesfoundation.org/how- } \\
\text { we-work/general-information/open-access-policy) }\end{array}$ \\
\hline & $\begin{array}{l}\text { Incitative } \\
\text { (encourageant } \\
\text { le partage) }\end{array}$ & $\begin{array}{l}\text { Le financeur encourage les chercheurs à partager sans } \\
\text { que cela ne soit une condition obligatoire pour être } \\
\text { financé. Il peut pour cela mettre en place différents } \\
\text { incitatifs. }\end{array}$ \\
\hline \multirow[t]{2}{*}{$\begin{array}{l}\text { Existence } \\
\text { d'incitatifs }\end{array}$} & $\begin{array}{l}\text { Lignes } \\
\text { budgétaires }\end{array}$ & $\begin{array}{l}\text { Le financeur met à disposition des chercheurs des lignes } \\
\text { financières dédiées à la préparation et au stockage des } \\
\text { données. Le partage des données impose en effet une } \\
\text { préparation pour rendre les données réutilisables et } \\
\text { garantir la sécurité des patients. }\end{array}$ \\
\hline & Autre incitatif & $\begin{array}{l}\text { Pour cette catégorie, nous acceptions tout type d'incitatif. } \\
\text { Le financeur peut par exemple conditionner le } \\
\text { financement de projets ultérieurs au partage des données } \\
\text { lors de financements antérieurs. }\end{array}$ \\
\hline $\begin{array}{l}\text { Existence de } \\
\text { sanctions en } \\
\text { cas de non- } \\
\text { partage }\end{array}$ & & $\begin{array}{l}\text { Pour cette catégorie, nous acceptions tout type de } \\
\text { sanction. Par exemple, le financeur pourrait décider de ne } \\
\text { pas verser la dernière part de budget en cas de non } \\
\text { partage. }\end{array}$ \\
\hline \multirow{2}{*}{$\begin{array}{l}\text { Existence de } \\
\text { guidelines pour } \\
\text { un mode } \\
\text { spécifique de } \\
\text { partage de } \\
\text { données }\end{array}$} & $\begin{array}{l}\text { Partage sur } \\
\text { requête }\end{array}$ & $\begin{array}{l}\text { Ce mode de partage se fait par contact (par exemple } \\
\text { email) entre les chercheurs souhaitant les données et les } \\
\text { chercheurs disposant des données. }\end{array}$ \\
\hline & $\begin{array}{l}\text { Partage sur une } \\
\text { plateforme } \\
\text { dédiée }\end{array}$ & $\begin{array}{l}\text { Les données sont déposées sur de larges plateformes de } \\
\text { partage de données comme Vivli, the Yale open data } \\
\text { access (YODA) project ou encore clinical study data } \\
\text { request. }\end{array}$ \\
\hline \multirow{2}{*}{$\begin{array}{l}\text { Type de } \\
\text { données à } \\
\text { partager }\end{array}$} & $\begin{array}{l}\text { Données } \\
\text { individuelles }\end{array}$ & $\begin{array}{l}\text { Les données individuelles des patients issus des essais } \\
\text { cliniques sont disponibles au partage. }\end{array}$ \\
\hline & $\begin{array}{l}\text { Code } \\
\text { statistique et } \\
\text { autre type de } \\
\text { données }\end{array}$ & $\begin{array}{l}\text { Le partage des données va au-delà des données } \\
\text { individuelles : le code statistique, les rapports d'études, le } \\
\text { plan d'analyse statistique, le protocole sont aussi } \\
\text { concernés par la politique de partage. }\end{array}$ \\
\hline \multirow{2}{*}{$\begin{array}{l}\text { Restrictions } \\
\text { vers un partage } \\
\text { de données aux } \\
\text { chercheurs } \\
\text { uniquement }\end{array}$} & $\begin{array}{l}\text { Existence de } \\
\text { restrictions }\end{array}$ & Le partage des données ne se fait qu'entre chercheurs. \\
\hline & $\begin{array}{l}\text { Aucune } \\
\text { restriction }\end{array}$ & $\begin{array}{l}\text { Le partage des données peut se faire avec des chercheurs, } \\
\text { mais aussi avec tout autre acteur, comme par exemple des } \\
\text { associations de patients, des journalistes, des citoyens, } \\
\text { des industriels... }\end{array}$ \\
\hline
\end{tabular}

Tableau 2. Caractéristiques des financeurs d'ECR français ayant une politique de partage de données par rapport à ceux qui n'en ont pas.

Les résultats sont sous forme de nombre (pourcentage) pour les variables qualitatives et de médiane (interquartile range) pour les variables quantitatives. 


\begin{tabular}{|l|c|c|c|}
\hline & $\begin{array}{l}\text { Financeurs inclus } \\
\mathbf{N = 3 1 ( 1 0 0 )}\end{array}$ & $\begin{array}{l}\text { Financeurs ayant une } \\
\text { politique de partage } \\
\text { de données } \\
\mathbf{N = 9}(\mathbf{2 9})\end{array}$ & $\begin{array}{l}\text { Financeurs } \\
\text { n'ayant pas de } \\
\text { politique de } \\
\text { partage de } \\
\text { données } \\
\mathbf{N = 2 2}(\mathbf{7 1})\end{array}$ \\
\hline $\begin{array}{l}\text { Enregistrement } \\
\text { obligatoire des ECR } \\
\text { financés }\end{array}$ & $12(39)$ & $6(67)$ & $6(27)$ \\
\hline $\begin{array}{l}\text { Publication } \\
\text { obligatoire des } \\
\text { résultats des ECR } \\
\text { financés }\end{array}$ & $13(42)$ & $6(67)$ & $7(32)$ \\
\hline $\begin{array}{l}\text { Nombre d'appels } \\
\text { d'offres médian en } \\
\text { 2018 }\end{array}$ & $2(1-5)$ & $2(1.5-4)$ & $2(1-5)$ \\
\hline $\begin{array}{l}\text { Volume financier } \\
\text { médian des AO en } \\
\mathbf{2 0 1 8}\end{array}$ & $(362500-6590000)$ & $(253000-1000000)$ & $\begin{array}{c}400000-7620 \\
000)\end{array}$ \\
\hline
\end{tabular}

ECR : essai contrôlé randomisé ; AO : appels d'offres

Tableau 3. Volume financier et nombre d'appels d'offres des dix plus gros financeurs, déclarés lors de notre enquête

\begin{tabular}{|l|l|l|l|}
\cline { 2 - 4 } \multicolumn{1}{l|}{} & $\begin{array}{l}\text { Volume financier } \\
\text { (euros) }\end{array}$ & $\begin{array}{l}\text { Nombre } \\
\text { d'appels à } \\
\text { projets }\end{array}$ & $\begin{array}{l}\text { Politique de partage } \\
\text { de données }\end{array}$ \\
\hline $\begin{array}{l}\text { Agence nationale de la } \\
\text { recherche }\end{array}$ & 600000000 & 50 & Non \\
\hline $\begin{array}{l}\text { Direction générale de } \\
\text { l'offre de soins }\end{array}$ & 130000000 & 8 & Non \\
\hline $\begin{array}{l}\text { Ligue contre le cancer } \\
\text { Vaincre la } \\
\text { mucoviscidose }\end{array}$ & 36500000 & 9 & $\begin{array}{l}\text { Encourage le partage } \\
\text { de données }\end{array}$ \\
\hline $\begin{array}{l}\text { Institut national contre } \\
\text { le cancer }\end{array}$ & 22000000 & 1 & Non \\
\hline $\begin{array}{l}\text { Hospices civils de Lyon } \\
\text { Fondation APICIL }\end{array}$ & 12000000 & 4 & Non \\
\hline GIRCI EST & 7620000 & 150 & Non \\
\hline $\begin{array}{l}\text { GIRCI GRAND } \\
\text { OUEST }\end{array}$ & 28000000000000 & 4 & Non \\
\hline Fondation ARSEP & 2280000 & 3 & Non \\
\hline
\end{tabular}




\begin{tabular}{|c|c|}
\hline & $\begin{array}{l}\text { Financeurs ayant une politique de } \\
\text { partage de données } \\
\qquad \mathbf{N}=\mathbf{9}(28)\end{array}$ \\
\hline $\begin{array}{r}\text { Type de partage de données } \\
\text { Encourage le partage } \\
\text { Oblige le partage }\end{array}$ & $\begin{array}{c}8(89) \\
1(11)\end{array}$ \\
\hline $\begin{array}{r}\text { Lignes budgétaires allouées au partage } \\
\mathrm{Oui}^{2} \\
\text { Non }\end{array}$ & $\begin{array}{l}5(56) \\
4(44)\end{array}$ \\
\hline $\begin{array}{r}\text { Récompenses en cas de partage } \\
O u i^{3} \\
\text { Non }\end{array}$ & $\begin{array}{l}3(33) \\
6(67)\end{array}$ \\
\hline $\begin{array}{r}\text { Sanctions en cas de non-partage de données: } \\
\text { Oui } \\
\text { Non }\end{array}$ & $9(100)$ \\
\hline $\begin{array}{r}\text { Mise en place de guidelines dédiées au } \mathrm{PD}: \\
\mathrm{Oui}^{4} \\
\mathrm{Non}\end{array}$ & $\begin{array}{l}6(67) \\
3(33)\end{array}$ \\
\hline $\begin{array}{r}\text { Mode de partage recommandé : } \\
\text { Dont sur requête } \\
\text { Dont sur des plateformes } \\
\text { Dont les deux }\end{array}$ & $\begin{array}{c}3(33) \\
1 \\
1 \\
1\end{array}$ \\
\hline $\begin{array}{r}\text { Types de données partagées : } \\
D I P^{8} \\
\text { DIP }+ \text { Protocoles et amendements }{ }^{9}\end{array}$ & $\begin{array}{l}1(33) \\
2(67)\end{array}$ \\
\hline Partage de données uniquement aux chercheurs ${ }^{4}$ & $3(33)$ \\
\hline
\end{tabular}

Tableau 4. Caractéristiques des politiques de partage de données

Association pour la Recherche sur la sclérose latérale amyothropique ${ }^{2,4,5,9}$, Société francophone d'arthroscopie ${ }^{2}$, Association Hubert Gouin ${ }^{2,3}$, La ligue contre le cancer ${ }^{2,3}$, Association française d'urologie e $^{2,3,4,7,9}$, GIRCI Nord-Ouest ${ }^{1}$, Institut national de la santé et de la recherche médicale, Société française de cardiologie, Institut de recherche sur la moëlle épinière ${ }^{4,6,8}$ 